\title{
The Kernel Regression Method Based on the Multiplicative Model to Reduce Speckle for SAR image
}

\author{
Xinna Li ${ }^{\text {a }}$, Ke Chen ${ }^{b}$ \\ Information Engineering University, Zhengzhou 450001, China \\ alixinna22@126.com, bchenke83@126.com
}

\begin{abstract}
Keywords: kernel regression; maximum likelihood; multiplicative model; SAR image; speckle; Abstract. Considering the multiplicative model for speckle noises, the kernel regression base on multiplicative model is built as the maximum likelihood estimation. Then, the gray similarity measure is put up forward based on the Gamma distribution, so the speckle reduction based on the multiplicative model for SAR image is obtained. Experimental results show that the proposed method can reduce speckle noise while preserving targets and edges.
\end{abstract}

\section{Introduction}

Kernel methods have been widely used in the computer field, especially in the settlement of pattern detection and pattern recognition problems[1]. There are some applications of kernel methods in the area of image and video processing already, but these methods have not been widely used, especially in SAR image processing. Compared to other parameter estimation methods, kernel regression is a non-parametric estimation method [2,3], which is based on the data itself to define the structure of the model, and this advantage ensures kernel regression method broad scope of application.

In fact, some technologies in image processing area are closely related with kernel regression, for example, the second order convolution, the bilateral filter, the edge interpolation and mobile least squares. But all the kernel regression methods and their improving forms are built on the additive noise model. They may fail if these methods are directly used to remove the multiplicative noise.

\section{Kernel regression based on the additive model}

The classical kernel regression method based on the additive model is as follows:

$$
y_{i}=f\left(x_{i}\right)+\varepsilon_{i}, i=1,2, \cdots, P
$$

Where $y_{i}$ are measurements, $f\left(x_{i}\right)$ is the (hitherto unspecified) regression function (i.e. an unknown image) to be estimated, and $\varepsilon_{i}$ are independent and identically distributed zero mean noise values. As the specific form of $f\left(x_{i}\right)$ is unspecified, in order to estimate the value of the function at any point $\mathbf{x}$ given by the data, one can trust in a generic, local expansion of the function about this point. Specifically, if $\mathbf{x}$ is near the sample at $\mathbf{x} i$, we have the $N$-term Taylor series.

$$
\begin{aligned}
f\left(x_{i}\right) & \approx f(x)+f^{\prime}(x)\left(x_{i}-x\right)+\frac{1}{2} f^{\prime \prime}(x)\left(x_{i}-x\right)^{2}+\cdots+\frac{1}{N !} f^{(N)}(x)\left(x_{i}-x\right)^{N} \\
& =\beta_{0}+\beta_{1}\left(x_{i}-x\right)+\beta_{2}\left(x_{i}-x\right)^{2}+\cdots+\beta_{N}\left(x_{i}-x\right)^{N}
\end{aligned}
$$

So $\varepsilon_{i}=y_{i}-f\left(x_{i}\right) \approx y_{i}-\beta_{0}-\beta_{1}\left(x_{i}-x\right)-\beta_{2}\left(x_{i}-x\right)^{2}-\cdots-\beta_{N}\left(x_{i}-x\right)^{N}$

A least-squares formulation capturing this idea is to solve the following optimization problem:

$$
\min \sum_{i=1}^{P}\left[y_{i}-\beta_{0}-\beta_{1}\left(x_{i}-x\right)-\beta_{2}\left(x_{i}-x\right)^{2}-\cdots-\beta_{N}\left(x_{i}-x\right)^{N}\right]^{2} \frac{1}{h} K\left(\frac{x_{i}-x}{h}\right)
$$

\section{Kernel regression based on the Multiplicative Model for SAR Image}

According to the statistical characteristics of the echo signal, the multiplicative model for SAR image is built as following[4]

$$
y_{i}=f\left(x_{i}\right) \bullet n_{i} \quad x_{i}=\left[x_{1 i}, x_{2 i}\right]^{\mathrm{T}}, i=1,2, \cdots, P
$$


Where $\left[x_{1 i}, x_{2 i}\right]$ are the azimuth coordinate and distance coordinate, $y_{i}$ is the according density of image polluted by speckle noise, $f\left(x_{i}\right)$ is radar cross section of ground target, $n_{i}$ is the speckle noise, which follows the Gamma distribution with mean 1 and variance $1 / L, L$ is the ENL(equivalent number looks), $P$ is the number of samples.

From the view of regression theory, $f\left(x_{i}\right)$ is the regression function, and $y_{i}$ is the observation at the coordinate point $x_{i}$. Correspondingly, the local expansion of the regression function is given by

$$
\begin{aligned}
f\left(x_{i}\right) & \approx f(x)+\{\nabla f(x)\}^{\mathrm{T}}\left(x_{i}-x\right)+\frac{1}{2}\left(x_{i}-x\right)^{\mathrm{T}}\left\{\nabla^{2} f(x)\right\}\left(x_{i}-x\right) \\
& =f(x)+\{\nabla f(x)\}^{\mathrm{T}}\left(x_{i}-x\right)+\frac{1}{2} \operatorname{vec}^{\mathrm{T}}\left\{\nabla^{2} f(x)\right\} \operatorname{vec}\left\{\left(x_{i}-x\right)\left(x_{i}-x\right)^{\mathrm{T}}\right\} \\
& =\beta_{0}+\beta_{1}^{\mathrm{T}}\left(x_{i}-x\right)+\frac{1}{2} \beta_{2}^{\mathrm{T}} \operatorname{vec}\left\{\left(x_{i}-x\right)\left(x_{i}-x\right)^{\mathrm{T}}\right\}
\end{aligned}
$$

where $\nabla f(x)$ and $\nabla^{2} f(x)$ are respectively the $2 \times 1$ gradient operator and $2 \times 2$ Hessian operator, and vec is the vectorization operator, which lexicographically orders a matrix into a vector, and

$$
\begin{aligned}
& \beta_{0}=f(x), \beta_{1}=\nabla f(x)=\left[\frac{\partial f(x)}{\partial x_{1}}, \frac{\partial f(x)}{\partial x_{2}}\right]^{\mathrm{T}} \triangleq\left[\beta_{11}, \beta_{12}\right]^{\mathrm{T}}, \\
& \beta_{2}=\frac{1}{2}\left[\frac{\partial^{2} f(x)}{\partial x_{1}^{2}}, \frac{\partial^{2} f(x)}{\partial x_{1} \partial x_{2}}, \frac{\partial^{2} f(x)}{\partial x_{2}^{2}}\right]^{\mathrm{T}} \triangleq \frac{1}{2}\left[\beta_{21}, \beta_{22}, \beta_{23}\right]^{\mathrm{T}} .
\end{aligned}
$$

According to the model (1) and the Taylor expansion (4), we have

$$
n_{i}=\frac{y_{i}}{f\left(x_{i}\right)} \approx \frac{y_{i}}{\beta_{0}+\beta_{1}^{\mathrm{T}}\left(x_{i}-x\right)+\frac{1}{2} \beta_{2}^{\mathrm{T}} \operatorname{vec}\left\{\left(x_{i}-x\right)\left(x_{i}-x\right)^{\mathrm{T}}\right\}}=\frac{y_{i}}{m(\beta)}
$$

Where $m(\beta) \triangleq \beta_{0}+\beta_{1}^{\mathrm{T}}\left(x_{i}-x\right)+\frac{1}{2} \beta_{2}^{\mathrm{T}} \operatorname{vec}\left\{\left(x_{i}-x\right)\left(x_{i}-x\right)^{\mathrm{T}}\right\}$. Then $n_{i}$ is can be regarded as the sample of $n$, so the weighted sample likelihood function is obtained as

$$
L\left(\beta_{0}, \beta_{1}, \beta_{2}\right)=p\left(n_{1}, n_{2}, \cdots, n_{P}\right)=\prod_{i=1}^{P}\left[\frac{L^{L}}{\Gamma(L)} n_{i}^{L-1} \exp \left\{-L n_{i}\right\}\right]^{w_{i}}
$$

Where $w_{i}$ measures the reliability of $n_{i}$.

From the according Logarithmic likelihood function, the optimization objective function becomes

$$
\min _{\beta_{0}, \beta_{1}, \beta_{2}} \sum_{i=1}^{P} \frac{w_{i} y_{i}}{\left[\beta_{0}+\beta_{1}^{\mathrm{T}}\left(x_{i}-x\right)+\frac{1}{2} \beta_{2}^{\mathrm{T}} \operatorname{vec}\left\{\left(x_{i}-x\right)\left(x_{i}-x\right)^{\mathrm{T}}\right\}\right]}+\sum_{i=1}^{P} w_{i} \ln \left[\beta_{0}+\beta_{1}^{\mathrm{T}}\left(x_{i}-x\right)+\frac{1}{2} \beta_{2}^{\mathrm{T}} \operatorname{vec}\left\{\left(x_{i}-x\right)\left(x_{i}-x\right)^{\mathrm{T}}\right\}\right]
$$

Respectively, the partial derivative with respect to $\beta_{0}, \beta_{1}, \beta_{2}$ are obtained and make them 0 ,ie.

$$
\left\{\begin{array}{l}
-\sum_{i=1}^{P} \frac{w_{i} y_{i}}{m^{2}(\beta)}+\sum_{i=1}^{P} \frac{w_{i}}{m(\beta)}=0 \\
-\sum_{i=1}^{P} \frac{w_{i} y_{i}\left(x_{i}-x\right)}{m^{2}(\beta)}+\sum_{i=1}^{P} \frac{w_{i}\left(x_{i}-x\right)}{m(\beta)}=0 \\
-\sum_{i=1}^{P} \frac{w_{i} y_{i} \operatorname{vec}\left\{\left(x_{i}-x\right)\left(x_{i}-x\right)^{\mathrm{T}}\right\}}{m^{2}(\beta)}+\sum_{i=1}^{P} \frac{w_{i} \operatorname{vec}\left\{\left(x_{i}-x\right)\left(x_{i}-x\right)^{\mathrm{T}}\right\}}{m(\beta)}=0
\end{array}\right.
$$

Then the numerical solution of $\beta_{0}$ is obtained by the steepest descent method from the nonlinear equation Group (5). And $\hat{\beta}_{0}$ is just the estimator of the regression function $f(x)$.

\section{gray similarity measure}

When the order of the local Taylor expansion of the regression function is zero, we have the 
approximation $f\left(x_{i}\right) \approx f(x)$. The noise observation is $n_{i}=\frac{Y_{i}}{f\left(x_{i}\right)} \approx \frac{Y_{i}}{f(x)}$ while the true noise is $n=\frac{Y}{f}$.

So the reliability $w_{i}$ is defined from the view of the probability as

$$
w_{i}=p\left(\frac{n_{i}}{n}\right) \approx p\left(\frac{Y_{i} / f}{Y / f}\right)=p\left(\frac{Y_{i}}{Y}\right)=\frac{L^{L}}{y \Gamma(L)}\left(\frac{y_{i}}{y}\right)^{L-1} \exp \left\{-\frac{L y_{i}}{y}\right\}
$$

\section{Parameter selection}

When computing (5) and (6), we need the parameter $L$. According to the model (1), for the smooth region, we have

$$
E(y)=E(f \bullet n)=f, D(y)=D(f \bullet n)=f^{2} D(n)=\frac{f^{2}}{L}
$$

So $L=\frac{f^{2}}{D(y)}=\frac{E^{2}(y)}{D(y)}$. Making the sample mean, sample variance instead of the mean and variance the of population, we get the moment estimation of $L$ is

$$
\hat{L}=\bar{y}^{2} / \hat{\sigma}^{2}(y)=(n-1) \bar{y}^{2} / \sum_{i=1}^{P}\left(y_{i}-\bar{y}\right)
$$

\section{Experimental results:}

In this section, a variety of simulations are carried out on the grey-scale Lena image to verify the performances of various restoration methods, including enhance Lee filter, enhance Frost filter, PM diffusion, classical kernel regression[3], logarithmic transform method (i.e., converting the multiplicative model to additive model by logarithmic transform, and then processing it by the classical kernel regression) and our method. The simulated images are corrupted by the noise with a wide range of noise variance varying from 0.01 to 0.1 . The mean square error (MSE) is used to evaluate the quantitative quality of the reconstructed images.

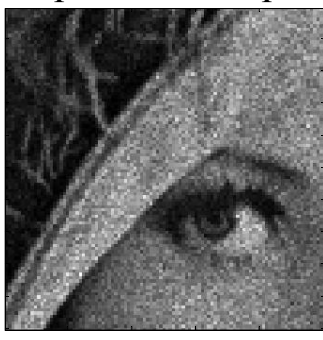

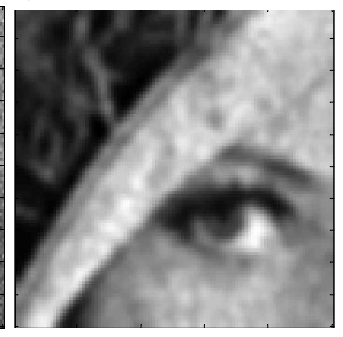

$d$
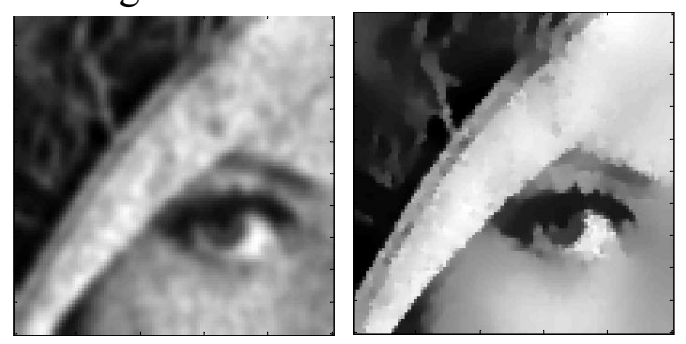

Fig. 1 Restoration results of different methods for Lena image corrupted with the variance of speckle noise 0.05.

$a$ Noisy image $b$ Classical kernel regression $c$ Logarithmic transform method $d$ Proposed method

Fig.1shows the results of different approaches in restoring corrupted Lena image with the variance of the speckle noise 0.05 . Obviously, our approach performs significantly better than the other methods. The comparison of restoration results in MSE and ENL for the reference image corrupted with various noise variances is shown in Fig.2. Apparently, the performance of our method is better than other methods for most cases. 
In addition, in Fig. 3 the results of the previous filters on MiniSAR image from Sandia laboratory are reported. As can be seen from Fig.3, even on real SAR images, the proposed method manages to fully remove speckle without smoothing the finest details.
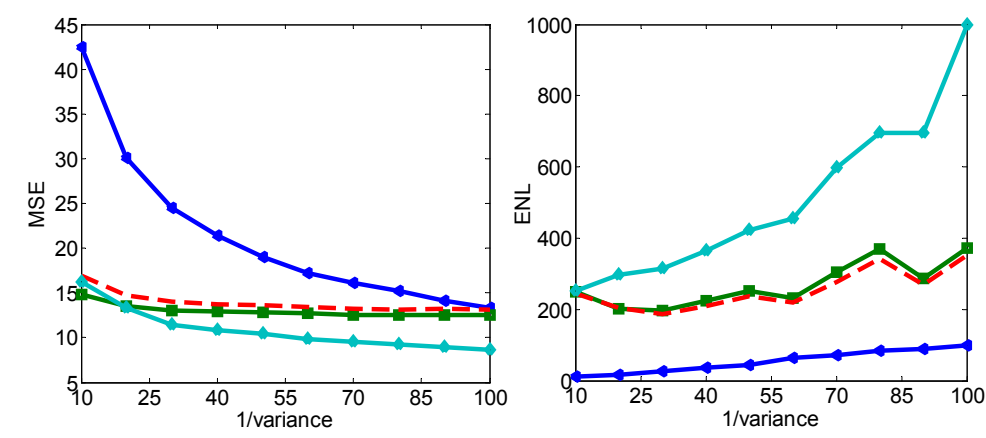

$\rightarrow$ original image $=$ - classical kernel regression - -logarithmic transform method $\rightarrow-$ proposed method

Fig. 2 Comparison of restoration results in MSE and ENL

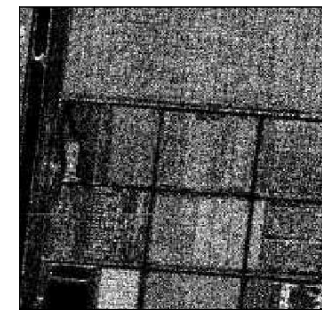

$a$

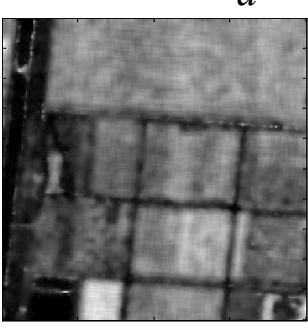

$d$

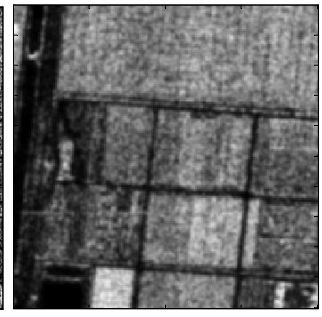

b

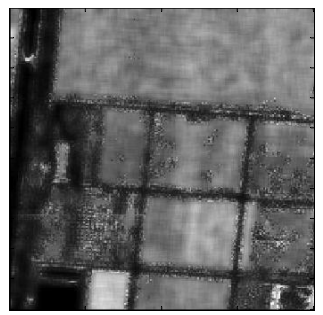

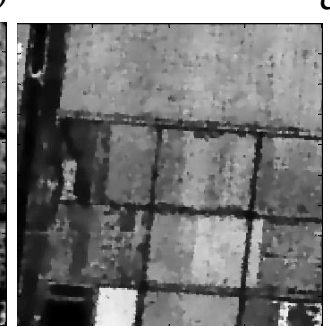

$e$ $c$

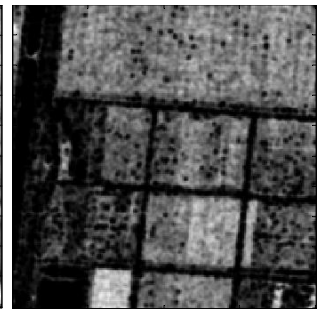

Fig. 3Restoration results of different methods for MiniSAR image. $a$ MiniSAR image $b$ Enhance Lee filter $c$ Enhance Frost filter

$d$ Classical kernel regression $e$ Logarithmic transform method $f$ Proposed method

\section{Conclusions}

In the paper, the kernel regression based on multiplicative model is built as the maximum likelihood estimation. The significant benefit of the proposed method is that the structure of the model is established by the data itself only. Experimental results show that the proposed method can reduce speckle noise while preserving targets and edges.

\section{References}

1. Eubank R L: 'Nonparametric Regression and Spline Smoothing' (Marcel Dekker, New York, USA, 1999)

2. Hiroyuki Takeda: 'Locally Adaptive Kernel Regression Methods for Multi-dimensional Signal Processing', Electrical Engineering, UC Santa Cruz, September 2010 
3. Hiroyuki Takeda. Sina Farsiu, and Peyman Milanfar: 'Kernel Regression for Image Processing and Reconstruction', IEEE Trans. Signal Process., 2007, 16, (2), pp. 349-366, doi: 10.1109/TIP.2006.888330

4. Goodman J W: 'Some Fundamental Properties of Speckle'. J.Opt.Soc.Am., 1976, 66(11): pp.1145-1150. 